\title{
Age of obesity onset, cumulative obesity exposure over early adulthood and risk of type 2 diabetes
}

\author{
Juhua Luo ${ }^{1}$ - Allison Hodge ${ }^{2,3} \cdot$ Michael Hendryx ${ }^{4}$ - Julie E. Byles ${ }^{5}$ \\ Received: 10 October 2019 / Accepted: 31 October 2019 / Published online: 20 December 2019 \\ (C) Springer-Verlag GmbH Germany, part of Springer Nature 2019
}

\begin{abstract}
Aims/hypothesis Obesity is a risk factor for type 2 diabetes, yet little is known about how timing and cumulative exposure of obesity are related to disease risk. The aim of this study was to examine the associations between BMI trajectories, age of onset of obesity and obese-years (a product of degree and duration of obesity) over early adulthood and subsequent risk of type 2 diabetes. Methods Women aged 18-23 years at baseline $(n=11,192)$ enrolled in the Australian Longitudinal Study on Women's Health (ALSWH) in 1996 were followed up about every 3 years via surveys for up to 19 years. Self-reported weights were collected up to seven times. Incident type 2 diabetes was self-reported. A growth mixture model was used to identify distinct BMI trajectories over the early adult life course. Cox proportional hazards regression models were used to examine the associations between trajectories and risk of diabetes.

Results One hundred and sixty-two (1.5\%) women were newly diagnosed with type 2 diabetes during a mean of 16 years of follow-up. Six distinct BMI trajectories were identified, varying by different initial BMI and different slopes of increase. Initial BMI was positively associated with risk of diabetes. We also observed that age at onset of obesity was negatively associated with risk of diabetes (HR 0.87 [95\% CI $0.79,0.96]$ per 1 year increment), and number of obese-years was positively associated with diabetes ( $p$ for trend $<0.0001$ ).

Conclusions/interpretation Our data revealed the importance of timing of obesity, and cumulative exposure to obesity in the development of type 2 diabetes in young women, suggesting that preventing or delaying the onset of obesity and reducing cumulative exposure to obesity may substantially lower the risk of developing diabetes.
\end{abstract}

Keywords Age of obesity onset $\cdot$ Cumulative obesity $\cdot$ Diabetes $\cdot$ Obese-years $\cdot$ Obesity $\cdot$ Type 2 diabetes $\cdot$ Weight trajectory

Abbreviations

BIC Bayesian information criterion

Juhua Luo

juhluo@indiana.edu

1 Department of Epidemiology and Biostatistics, School of Public Health, Indiana University, 1025 E 7th Street,

Bloomington, IN 47405, USA

2 Cancer Epidemiology Division, Cancer Council Victoria, Melbourne, VIC, Australia

3 Centre for Epidemiology and Biostatistics, Melbourne School of Population and Global Health, University of Melbourne, Parkville, VIC, Australia

4 Department of Environmental and Occupational Health, School of Public Health, Indiana University, Bloomington, IN, USA

5 Research Centre for Generational Health and Ageing, University of Newcastle, Newcastle, NSW, Australia
GMM Growth mixture model

MET Metabolic equivalents

hs-CRP High-sensitivity C-reactive protein

\section{Introduction}

The prevalence of type 2 diabetes has increased dramatically during the past several decades. Currently, more than 500 million adults are living with diabetes worldwide [1]. The increase in type 2 diabetes prevalence is closely related to the upsurge in obesity. At present, more than 2.1 billion people (nearly $30 \%$ of the world's population) are either obese (BMI $\left.\geq 30 \mathrm{~kg} / \mathrm{m}^{2}\right)$ or overweight $\left(25 \mathrm{~kg} / \mathrm{m}^{2} \leq \mathrm{BMI}<30 \mathrm{~kg} / \mathrm{m}^{2}\right)$ [2]. In 2017, almost two-thirds $(63 \%)$ of adults in Australia were overweight or obese, and $28 \%$ were obese [3]. 


\section{Research in context}

What is already known about this subject?

- Obesity is a risk factor for type 2 diabetes

What is the key question?

- Are earlier age at onset of obesity and greater cumulative obesity exposure over young adulthood associated with risk of type 2 diabetes?

What are the new findings?

- Age at onset of obesity was negatively associated with risk of type 2 diabetes in a cohort of women aged 18-23 years at baseline

- Obese-years (a measure of cumulative obesity exposure) was positively associated with type 2 diabetes in this cohort

How might this impact on clinical practice in the foreseeable future?

- Our data reveal the importance of timing of, and cumulative exposure to, obesity in the development of type 2 diabetes in young women, suggesting that preventing or delaying the onset of obesity and reducing cumulative exposure may substantially lower the risk of developing diabetes

Although obesity is a well-established risk factor for type 2 diabetes [4], little is known about the relationships between age of onset of obesity and cumulative exposure to obesity and risk of diabetes, especially among young adults. In most previous studies, exposures (obesity status or weight change) have relied on only one or two measures of weight [5]. As a result, researchers have been unable to account for the dynamic changes of weight over time, or to assess whether timing of weight change over the life course makes a difference to the risk of developing type 2 diabetes.

A few studies have examined the association between the duration of general or central obesity and the risk of diabetes but results are mixed [6-9]. Some studies reported that duration of obesity was associated with the risk of diabetes independently of baseline BMI [7, 9], while others reported that the association between diabetes and duration of obesity was largely determined by current BMI $[6,8]$.

Obese-years is a construct that accounts for both how long a person has been obese and the magnitude of their obesity (no. of BMI units above $30 \mathrm{~kg} / \mathrm{m}^{2} \times$ no. of years at that BMI) and has been shown to be a better predictor of diabetes risk than duration of obesity or level of BMI alone [10]. However, few studies have examined the relationship between the cumulative exposure to obesity and risk of diabetes.

We used data from the Australian Longitudinal Study on Women's Health (ALSWH) to identify BMI trajectories over the early adult life course. We then examined the relationship between distinct BMI trajectories and risk of type 2 diabetes. Further, we investigated the associations between timing of obesity onset, obese-years and type 2 diabetes. We hypothesise that earlier age at onset of obesity and more obese-years over follow-up would be more strongly associated with type 2 diabetes risk than $\mathrm{BMI}$ alone.

\section{Methods}

\section{ALSWH}

The ALSWH is a longitudinal population-based study of Australian women that was launched in 1996. It was designed to examine the relationships between biological, psychological, social and lifestyle factors and women's physical health, emotional wellbeing, and their use of and satisfaction with healthcare [11]. This study recruited a nationally representative sample of more than 40,000 women in three age cohorts, born in 1921-1926 (aged 70-75 years at baseline), 19461951 (aged 45-50 years) and 1973-1978 (aged 18-23 years). Details of the design, recruitment, implementation and progress of the ALSWH study are described elsewhere [11-15]. The study was approved by the Universities of Newcastle and Queensland Ethics Review Committees. All participants provided written informed consent.

\section{Study population}

The current study was based on data from the 1973-1978 birth cohort (aged 18-23 years at recruitment, $n=14,247$ ). Participants were excluded from the analysis for the following reasons: they reported a history of diabetes at enrolment or reported having type 1 diabetes during follow-up ( $n=247)$; they answered only the baseline survey $(n=2309)$; they had missing values for major covariates at baseline including physical activity, income and stress $(n=133)$; or they had no BMI data $(n=366)$. After exclusions, 11,192 women remained in the analytical cohort. 


\section{Follow-up}

Participants completed survey 2 in 2000 (4 years after baseline) and have been followed up every 3 years thereafter up to 19 years and a total of seven survey administrations. Surveys were completed by mail, and an on-line option has been available since survey 6 in 2012. For the 1973-1978 cohort, the retention rate was low (69\%) from wave 1 to wave 2 , due to high levels of mobility and inability to contact participants at this stage, but then remained relatively stable thereafter with retention rates of $57-64 \%$ [15].

\section{Measurements}

Outcome: incidence of type 2 diabetes during follow-up Incident type 2 diabetes was defined via self-report of new doctor-diagnosed diabetes during follow-up. Self-reported diabetes in the ALSWH has been validated for middle-aged and older cohorts by comparing self-report data with administrative hospital records [16]. There was substantial agreement for diabetes $(\kappa>0.70)$ for both cohorts [16], indicating that self-reported diabetes in the ALSWH may be a reliable indicator of diagnosed diabetes.

Exposure: self-reported weight and height measures across surveys In the ALSWH, height was collected at baseline and weights were self-reported at baseline and at each follow-up survey. BMI was calculated as weight $(\mathrm{kg})$ divided by height (m) squared. Only measures of BMI before the date of diagnosis of type 2 diabetes were included in this analysis.

Accuracy of BMI estimated from self-reported height and weight has been validated in the ALSWH [17]. There was substantial agreement ( $84 \%$ ) between BMI categories derived from self-reported and measured height and weight data; agreement for healthy-weight women was $94 \%$. This suggests that the self-reported data on weight and height obtained from mailed surveys in the ALSWH can be used to estimate BMI with reasonable accuracy [17].

Covariates Participants' sociodemographic characteristics (age, educational attainment, income adequacy), lifestyle factors (physical activity, smoking, alcohol use), gestational diabetes history, and psychosocial factors (a stress score) measured at baseline were considered as potential confounders. Table 1 presents detailed information about covariate categories and scores. Income adequacy was assessed by an item asking 'how do you manage on the income you have available?' Physical activity data was collected by asking for the amount of time spent in the last week on different activities including walking briskly, moderate leisure activity and vigorous leisure activity and converting to metabolic equivalents (MET)/week (continuous). Perceived stress was measured continuously as a sum across items in specific life domains: own health, health of other family members, work/employment, living arrangements, study, money, relationship with parents, relationship with partner/spouse, relationships with children and relationship with other family members. The score has been demonstrated to be reliable and valid [15].

\section{Statistical analysis}

First, descriptive statistics were calculated. Second, BMI growth curves (trajectories) over time were examined. We fitted the data by using both linear and quadratic models and compared which of these models fit the data better by looking at whether the $z$ score test was significant for the quadratic growth factor mean. Since the quadratic growth factor mean was significant $(p<0.05)$, we used quadratic models for growth curves.

Third, a growth mixture model (GMM) was used to identify distinct BMI trajectories over time. The GMM allows modelling variables with partially missing data [18]. The optimal number of groups was assessed by the Bayesian information criterion (BIC) [19]. A lower BIC indicates better fit to the data. Once the best-fitting model was identified, participants were assigned to the trajectory groups to which their posterior membership probability was largest. Cox proportional hazards regression models were then used to evaluate the associations between the trajectories and risk of type 2 diabetes. Survival time was defined as time between baseline and the date of newly diagnosed diabetes, censoring due to missing data, death, or end of follow-up at survey 7, whichever came first. For women who reported newly diagnosed diabetes on a survey, we assumed the point of diagnosis was halfway through the period between that survey and the previous survey. The BMI trajectory would end at the previous survey. In the multivariate-adjusted models, potential confounders were included as covariates. We also examined relationships between sociodemographic characteristics, lifestyle and stress and BMI trajectories.

Fourth, since the GMM approach enables us to model BMI trajectories with random variation among individuals within a group, we allowed each individual to have her own BMI trajectory; namely, we predicted BMI trajectory (including intercept, slope and quadratic) for each individual. Using the predicted curve for each individual, we estimated the age of onset of obesity if any, degree of obesity (measured by difference in BMI above obesity $\left[\geq 30 \mathrm{~kg} / \mathrm{m}^{2}\right]$ ), and duration in years of obesity. Obese-years was estimated by calculating the AUC that was above $30 \mathrm{~kg} / \mathrm{m}^{2}$ by trapezoid rule [20]. Age of onset of obesity and obese-years were treated as time-varying covariates in Cox models.

We conducted a sensitivity analysis using baseline-only BMI categories to examine HRs for diabetes, with control 


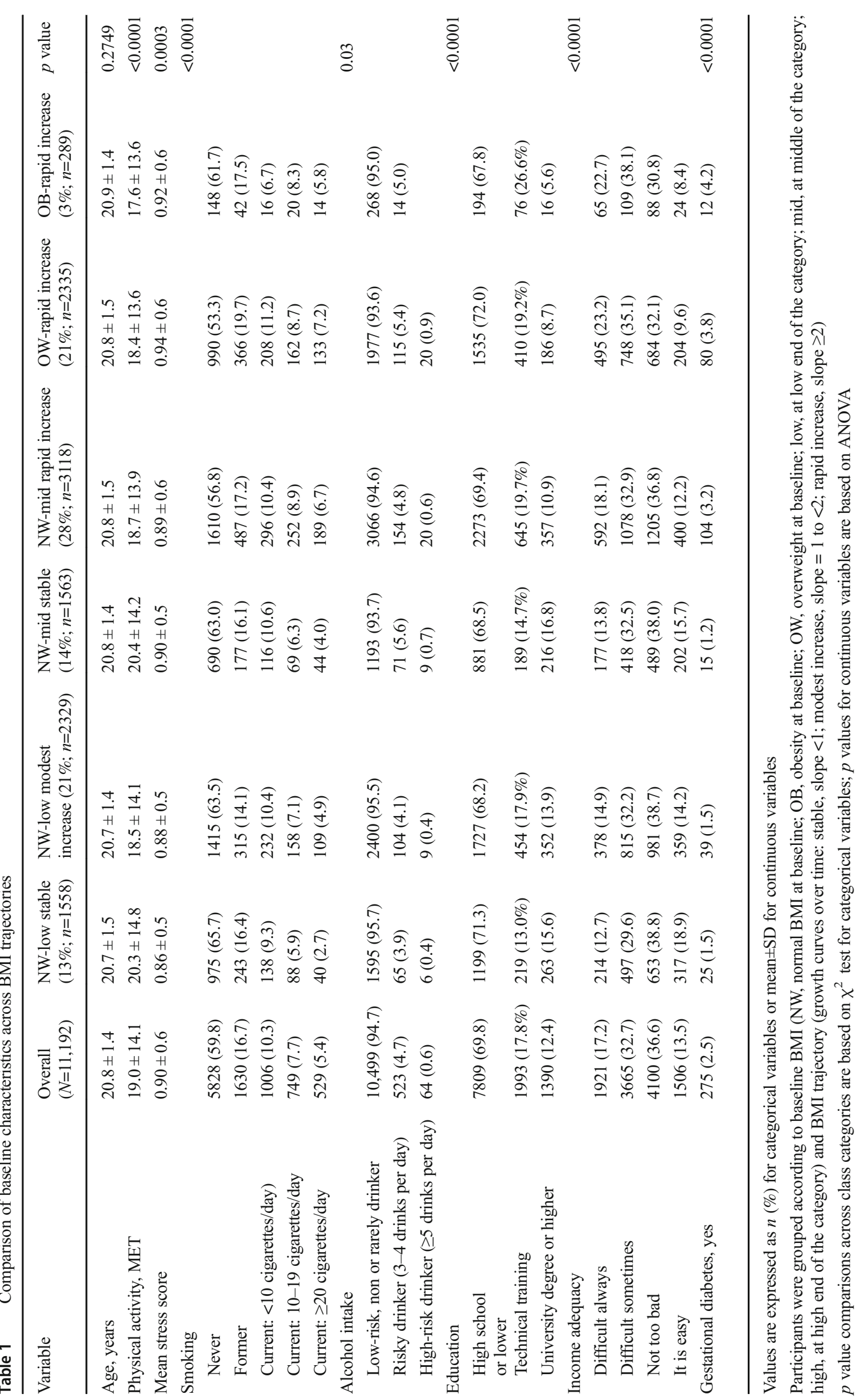


for the same covariates, to compare HRs to those obtained for weight trajectories.

Finally, we performed a sensitivity analysis by further adjusting for diet data from a food frequency questionnaire [21], including information about total energy intake, fibre intake, dietary glycaemic index and glycaemic load. Statistical analyses were performed using Mplus 8.2 [22] and SAS Version 9.4 (SAS Institute, Cary, NC, USA).

\section{Results}

Among 11,192 women who were free of diabetes at baseline, 162 women (1.5\%) developed type 2 diabetes during a mean of 16 years of follow-up. The mean BMI increased from $22.8 \mathrm{~kg} / \mathrm{m}^{2}$ at baseline to $26.9 \mathrm{~kg} / \mathrm{m}^{2}$ at survey 7 . The obesity prevalence rate increased from $6.5 \%$ at baseline to $25.7 \%$ at survey 7 , and the mean obesity-years was 8.4 years (SD 5.2) among the 2008 women who became obese.

We examined different quadratic models with varying number of classes (trajectories) from two to seven, and identified a six-class solution based on the lowest BIC value. We ranked the six classes (Fig. 1) based on their corresponding mean of BMI at baseline from low to high. We then categorised the four classes with baseline BMI $<25 \mathrm{~kg} / \mathrm{m}^{2}$ as normal weight, and labelled them according to their baseline BMI and slope: NW-low stable (slope <1); NW-mid stable; NW-low modest increase (slope 1 to $<2$ ); NW-rapid increase (slope $\geq 2$ ). The remaining two classes were overweight at baseline, with BMI 25 to $<30 \mathrm{~kg} / \mathrm{m}^{2}$ and with a rapid increase in BMI (OW-rapid increase), or obese (BMI $\geq 30 \mathrm{~kg} / \mathrm{m}^{2}$ ) with a rapid increase (OB-rapid increase).
In the study population, we observed that two classes had stable BMI trajectory over time (27\% of women), including NW-low stable and NW-mid stable classes; $21 \%$ of women with normal weight at baseline had modest BMI increase, and more than half of the women had a rapid increase of BMI over time, including those in the NW-rapid increase, OW-rapid increase and OB-rapid increase classes. The largest proportion (28\%) of women belonged to the NW-mid rapid increase class. The next most frequent categories were the OW-rapid increase group (21\%) and NW-low modest increase group (21\%). A small group (3\%) of women belonged to the OBrapid increase class, with initial BMI around $33 \mathrm{~kg} / \mathrm{m}^{2}$.

Table 1 shows baseline characteristics across the six BMI trajectories. Based on mean baseline BMI from low to high, compared with the lower ranked class, women in the higher ranked classes were more likely to be physically inactive, have higher stress scores, smoke more, be more risky drinkers, have lower education level, have more difficulty in managing on available income and were more likely to have a history of gestational diabetes. Baseline age across classes was not different (Table 1).

Estimated intercept, slope and quadratic parameters for each class are listed in Table 2. Specific growth trajectories are presented in Fig. 1. Compared with women in the NW-low stable class, two groups had significantly higher risk of type 2 diabetes (HR 4.75 [95\% CI 2.43, 9.28] for OW-rapid increase class; HR 10.06 [95\% CI 4.69, 21.58] for OB-rapid increase class] after adjustment for potential confounders. Initial BMI was significantly and positively associated with risk of type 2 diabetes. Slope was borderline significantly associated with the risk $(p=0.07)$ but the quadratic term was not associated with risk (Table 2).
Fig. 1 BMI trajectories over 19 years of follow-up. The estimated mean BMI in each trajectory is shown. Participants were grouped according to baseline BMI (NW, normal BMI; OB, obese; OW, overweight; low, at low end of the category; mid, at middle of the category) and BMI increase (growth curves over time: stable, slope $<1$; modest increase, slope 1 to $<2$; rapid increase, slope $\geq 2$ )

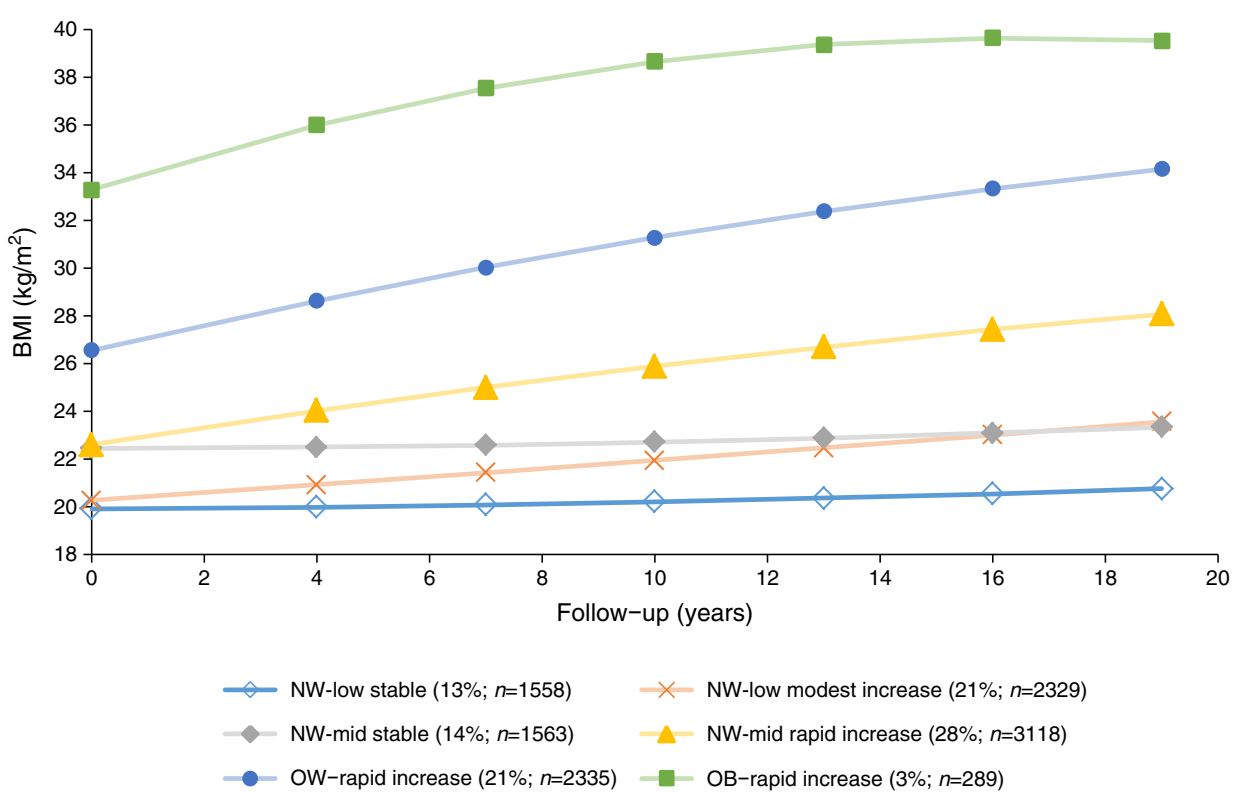


Table 2 Estimated means of intercept, slope and quadratic parameters for each BMI class, and HRs for type 2 diabetes incidence

\begin{tabular}{|c|c|c|c|c|c|}
\hline Class/parameter & No. of cases & Intercept & Slope & Quadratic & $\begin{array}{l}\text { Multivariable-adjusted model, } \\
\text { HR }(95 \% \mathrm{CI})^{\mathrm{a}}\end{array}$ \\
\hline \multicolumn{6}{|l|}{ Class } \\
\hline NW-low stable (13\% of study population) $(n=1558)$ & 10 & 19.92 & $0.13^{\mathrm{b}}$ & 0.16 & 1.00 \\
\hline NW-low modest increase ( $21 \%$ of study population) $(n=2329)$ & 21 & 20.27 & 1.61 & $0.07^{\mathrm{b}}$ & $1.34(0.63,2.85)$ \\
\hline NW-mid stable (14\% of study population) $(n=1563)$ & 11 & 22.46 & $0.03^{\mathrm{b}}$ & $0.23^{\mathrm{b}}$ & $1.37(0.50,3.22)$ \\
\hline NW-mid rapid increase ( $28 \%$ of study population) $(n=3118)$ & 32 & 22.61 & 3.72 & -0.44 & $1.49(0.73,3.04)$ \\
\hline OW-rapid increase ( $21 \%$ of study population) $(n=2335)$ & 67 & 26.56 & 5.51 & -0.79 & $4.75(2.43,9.28)$ \\
\hline OB-rapid increase (3\% of study population) $(n=289)$ & 21 & 33.28 & 7.69 & -2.32 & $10.06(4.69,21.58)$ \\
\hline \multicolumn{6}{|l|}{ Quadratic model parameter } \\
\hline Intercept & & & & & $1.12(1.09,1.15)$ \\
\hline Slope & & & & & $1.06(0.99,1.13)$ \\
\hline Quadratic & & & & & $0.97(0.83,1.14)$ \\
\hline
\end{tabular}

Participants were classed according to baseline BMI (NW, normal BMI at baseline; OB, obesity at baseline; OW, overweight at baseline; low, at low end of the category; mid, at middle of the category; high, at high end of the category) and BMI trajectory (growth curves over time: stable, slope $<1$; modest increase, slope $=1$ to $<2$; rapid increase, slope $\geq 2$ )

${ }^{a}$ Model adjusted for age (continuous), physical activity in (MET/week, continuous), smoking (never, former, current smoker [ $<10$ cigarettes/day, 10-19 cigarettes/day, $\geq 20$ cigarettes/day]), alcohol (low-risk, non or rarely drinker, risky drinker, high-risk drinker), education (high school or lower, technical training, university degree or higher), income adequacy (impossible, difficult always, difficult sometimes, not too bad, it is easy), stress score (continuous) and history of gestational diabetes (no, yes)

${ }^{\mathrm{b}}$ These estimates were not significant; $p<0.05$ for all other estimates

Among the 10,521 (94\%) women who were not obese at baseline, we observed that women who became obese during follow-up had a threefold (95\% CI 2.01, 4.50) risk of type 2 diabetes compared with women who did not become obese. There was a negative association between age at onset of obesity and risk of type 2 diabetes among women who became obese during follow-up (HR 0.87 [95\% CI 0.79, 0.96]) (Table 3). Compared with women who did not become obese during the follow-up, women who became obese and had obese-years of $<10,10$ to $<30$ and $\geq 30$ had an HR $(95 \%$ CI) of $2.18(1.25,3.81), 3.01(1.53,5.91)$ and $5.88(3.15,10.97)$, respectively ( $p$ for trend $<0.0001$ ) (Table 3 ).
The sensitivity analysis using only baseline BMI revealed that individuals with baseline obesity (BMI $\geq 30 \mathrm{~kg} / \mathrm{m}^{2}$ ) had an HR of 7.07 (95\% CI 4.73, 10.57), which is lower than the HR of 10.06 (95\% CI 4.69, 21.58) shown in Table 2 for individuals in the obese with rapid increase group. Similarly, individuals shown in Table 2 who were overweight with rapidly increasing BMI had an HR of 4.75 (95\% CI 2.43, 9.28), which was higher than the baseline-only overweight group (HR 2.33 [95\% CI 1.53, 3.55]).

A sensitivity analysis that further adjusted for the number of children and dietary intake (including total energy intake, fibre intake and dietary glycaemic index or glycaemic load) found similar results (data not shown).
Table 3 Associations between age at onset of obesity, obeseyears, and risk of type 2 diabetes incidence among 10,521 (94\%) women who were not obese at baseline

\begin{tabular}{lll}
\hline & Cases/population & HR $(95 \% \mathrm{CI})$ \\
\hline Became obese during follow-up & & \\
$\quad$ No & $79 / 8513$ & 1.00 \\
$\quad$ Yes & $38 / 2008$ & $3.01(2.01,4.50)$ \\
Age at onset of obesity among women who became obese & $38 / 2008$ & $0.87(0.79,0.96)^{\mathrm{a}}$ \\
Obese-years & & \\
Not obese & $79 / 8513$ & 1.00 \\
$<10$ & $15 / 916$ & $2.18(1.25,3.81)$ \\
10 to $<30$ & $10 / 494$ & $3.01(1.53,5.91)$ \\
$\geq 30$ & $13 / 598$ & $5.88(3.15,10.97)$ \\
$p$ for trend & $117 / 10,521$ & $<0.0001$ \\
\hline
\end{tabular}

${ }^{\mathrm{a}}$ For a 1 year increment in age of obesity onset 


\section{Discussion}

From this population-based prospective cohort of Australian young adult women with 19 years of follow-up, we identified six distinct BMI trajectories varying by initial BMI and slope. More than half of the women experienced a rapid BMI increase from early (18-23 years old) to middle adulthood (37-42 years old). Our data confirmed that BMI in young adulthood played an important role in the subsequent risk of developing type 2 diabetes during adulthood. We also observed that women who were non-obese at baseline but became obese during follow-up had a higher risk of type 2 diabetes relative to women who stayed non-obese; the younger the age at onset of obesity or the greater obese-years, the higher the risk of type 2 diabetes.

A few previous studies have investigated associations between BMI trajectories and diabetes incidence [23-26], diabetes-related metabolic markers, or inflammatory markers [27-30]. Findings from these studies indicate that risks for diabetes incidence, diabetes-related metabolic markers (i.e. glucose, insulin and HOMA-IR), or elevated hs-CRP (highsensitivity C-reactive protein) [23, 27-30] are associated with steeper weight gain trajectories, consistent with our findings. Two studies $[27,28]$ based on a cohort in a similar age group to our study also identified six similar trajectories and observed that a steeper weight gain trajectory was associated with higher risks of metabolic markers of diabetes or greater risk of elevated hs-CRP compared with more moderate weight grain trajectories. A recent study using repeated measurements of BMI from childhood to adulthood observed that the linear slope of BMI change between ages 10 and 19 years was positively associated with adult hyperglycaemia [31]. Another study based on retrospectively recalled weights from age 20 years and weight in middle-aged Japanese men and women found that long-term weight change slope was significantly associated with risk of type 2 diabetes [32]. That is, the steeper slope, the higher the risk of type 2 diabetes. In our study, the association between slope and risk of type 2 diabetes was only borderline significant. This may be due to the small number of women diagnosed with type 2 diabetes over time among this young cohort.

Our data indicated that baseline BMI among young women was significantly associated with risk of developing type 2 diabetes, with women in the overweight or obese BMI trajectories having significantly higher risk of type 2 diabetes compared with women in the NW-low stable group. The results highlight the importance of overweight or obesity in early adulthood as risk factors for adult diabetes, indicating that weight control starting before early adulthood is critical for reducing type 2 diabetes risk in later life.

In addition to initial BMI, we also observed that a higher number of obese-years was associated with higher risk of type 2 diabetes in a dose-response fashion. Unlike measurements of BMI at one point in time, measurement of obese-years account for the cumulative effect of obesity. A previous study compared different measures of obesity as predictors of diabetes risk and reported that obese-years was a better predictor for type 2 diabetes than duration of obesity or BMI values alone [10]. This may be because obese-years represent the cumulative damage to the body caused by obesity. It is well-accepted that excess adiposity can have deleterious metabolic effects such as increased insulin resistance or increased levels of proinflammatory cytokines [33, 34]. Prolonged duration of excess adiposity may result in additional metabolic changes, leading to the development of diabetes $[34,35]$. These results indicate the importance of both duration of obesity and the degree of obesity in the development of type 2 diabetes.

We also observed that older age at onset of obesity was associated with lower risk of type 2 diabetes when compared with younger age at onset. Our finding indicates that earlyonset obesity may be particularly deleterious for future diabetes risk in women. We only identified one study that examined age at onset of obesity in relation to the risk of type 2 diabetes [36]. This analysis, based on the Framingham Heart study, found that age of onset may be a useful, practical addition to current BMI in the elderly. One explanation for the finding of higher risk of type 2 diabetes among women who became obese at a younger age may be the prolonged duration of obesity, because women with early onset of obesity will likely also have longer duration of obesity. However, it is possible that obesity during young age may be more deleterious for insulin resistance and diabetes than obesity during older age $[37,38]$. Another study has also demonstrated that diabetes risk was particularly high in individuals who were obese as adolescents relative to those with adult-onset obesity [38]. These data highlight the need for early diabetes prevention efforts to prevent or delay the onset of obesity.

Strengths of the study include the long-term follow-up in a large prospective cohort. Several limitations deserve mention. First, weight was self-reported. There is evidence from a previous validation study that self-reported height and weight are reliable [17]; however, the reliability of self-reported weight is better for normal weight women than obese women. Thus, potential misclassification in trajectory assignment may still exist and the fact that the degree of under-reporting in weight is differential with respect to their true weight may lead to an overestimate of the associations between trajectories and diabetes risk. Second, although self-reported diabetes in the cohort has been validated for middle-aged and older cohorts and has been demonstrated to be a reliable indicator of diagnosed diabetes [16], a relatively larger degree of underdiagnosis in the young cohort is possible, which may bias our findings. For example, if diabetes is more likely to be under-diagnosed among obese women than non-obese women, our results may be under-estimated. On the other hand, if diabetes is less likely to be under-diagnosed for obese 
women than non-obese women our results may be over-estimated. The prevalence of diabetes we observed $(1.5 \%)$ was similar to self-reported data from the Australian Bureau of Statistics (ABS) 2014-15 National Health Survey, where the prevalence of diabetes was $1.5 \%$ among women aged 18 44 years [39]. Third, the low incidence of diabetes in our young study population during the follow-up period may be a concern for the Cox models, given these can produce unreliable estimates when the outcome is rare. Fourth, BMI data were only collected from baseline; thus, we were unable to calculate the timing of obesity for women who were already obese at baseline. These women had to be excluded from analyses for age at onset and obese-years. This may reduce study power despite the fact that these women accounted for only $6.5 \%$ of the study population. Another limitation is that our analysis does not consider the uncertainty of the classification rather than forcing individuals into their most likely trajectory patterns. Finally, the study was conducted among young Australian women. Caution should be taken when generalising our findings to other populations.

In conclusion, our data have shown that more than half of the women experienced a rapid BMI increase from early to middle adulthood, suggesting the importance of monitoring weight change over time. Persons who experience rapid weight gain are at increased diabetes risk independently of baseline BMI status. In particular, our data indicate the importance of timing of obesity and cumulative exposure to obesity measured by obese-years in relation to diabetes risk in young women. The results highlight the importance of preventing or delaying the onset of obesity and reducing cumulative exposure to obesity to substantially lower the risk of developing diabetes. We recommend that people self-monitor weight change over time, and that healthcare providers attend to weight change in addition to static weight as another risk factor for diabetes.

Acknowledgements We are grateful to the women who provided the survey data. The authors also thank G. Giles of the Cancer Epidemiology Division, Cancer Council Victoria, for permission to use the Dietary Questionnaire for Epidemiological Studies (Version 2), Melbourne: Cancer Council Victoria, 1996.

Data availability Data may be made available to collaborating researchers where there is a formal request to make use of the material. Permission to use the data must be obtained from the Data Access Committee of ALSWH (https://www.alswh.org.au/how-to-access-thedata/alswh-data).

Funding The research on which this paper is based was conducted as part of the ALSWH by the University of Queensland and the University of Newcastle. We are grateful to the Australian Government Department of Health for funding. This study was also supported by a Pilot and Feasibility Award within the Center for Diabetes and Metabolic Diseases (CDMD) at Indiana University School of Medicine, NIH/ NIDDK Grant Number P30 DK097512.

Duality of interest The authors declare that there is no duality of interest associated with this manuscript.
Contribution statement All the people listed as authors fulfil all the following three criteria: (1) substantial contributions to conception and design, acquisition of data, or analysis and interpretation of data; (2) drafting the article or revising it critically for important intellectual content; and (3) final approval of the version to be published. JL designed the study and drafted the manuscript. JEB acquired the data. JL is the guarantor of this work.

\section{References}

1. Kaiser AB, Zhang N, van der Pluijm W (2018) Global prevalence of type 2 diabetes over the next ten years (2018-2028). Diabetes 67(Suppl 1):202

2. Ng M, Fleming T, Robinson M et al (2014) Global, regional, and national prevalence of overweight and obesity in children and adults during 1980-2013: a systematic analysis for the Global Burden of Disease Study 2013. Lancet 384:766-781

3. Australian Institute of Health and Welfare (2017) A picture of overweight and obesity in Australia Available from https://www.aihw. gov.au/getmedia/172fba28-785e-4a08-ab37-2da3bbae40b8/aihwphe-216.pdf.aspx? inline=true. Accessed 10 Jun 2019

4. CDC (2019) Risk factors for diabetes. Available from https://www. cdc.gov/diabetes/basics/risk-factors.html. Accessed 6 May 2019

5. Kodama S, Horikawa C, Fujihara K et al (2014) Quantitative relationship between body weight gain in adulthood and incident type 2 diabetes: a meta-analysis. Obes Rev 15:202-214

6. Bouchard DR, Porneala B, Janssen I et al (2013) Risk of type 2 diabetes and cumulative excess weight exposure in the Framingham Offspring Study. J Diabetes Complications 27:214218

7. Abdullah A, Stoelwinder J, Shortreed S et al (2011) The duration of obesity and the risk of type 2 diabetes. Public Health Nutr 14(1): 119-126. https://doi.org/10.1017/S1368980010001813

8. Hu Y, Bhupathiraju SN, de Koning L, Hu FB (2014) Duration of obesity and overweight and risk of type 2 diabetes among US women. Obesity (Silver Spring) 22(10):2267-2273. https://doi. org/10.1002/oby.20851

9. Reis JP, Hankinson AL, Loria CM et al (2013) Duration of abdominal obesity beginning in young adulthood and incident diabetes through middle age: the CARDIA study. Diabetes Care 36(5): 1241-1247. https://doi.org/10.2337/dc12-1714

10. Abdullah A, Wolfe R, Mannan H, Stoelwinder JU, Stevenson C, Peeters A (2012) Epidemiologic merit of obese-years, the combination of degree and duration of obesity. Am J Epidemiol 176(2): 99-107. https://doi.org/10.1093/aje/kwr522

11. Brown WJ, Bryson L, Byles JE et al (1998) Women's Health Australia: recruitment for a national longitudinal cohort study. Women Health 28:23-40

12. Brown WJ, Dobson AJ, Bryson L, Byles JE (1999) Women's Health Australia: on the progress of the main cohort studies. J Womens Health Gend Based Med 8(5):681-688. https://doi.org/ 10.1089/jwh.1.1999.8.681

13. Dobson AJ, Hockey R, Brown WJ et al (2015) Cohort Profile Update: Australian Longitudinal Study on Women's Health. Int J Epidemiol 44:1547 1547a-1547f

14. Lee C, Dobson AJ, Brown WJ et al (2005) Cohort Profile: the Australian Longitudinal Study on Women's Health. Int J Epidemiol 34(5):987-991. https://doi.org/10.1093/ije/dyi098

15. Women's Health Australia (2019) Australian Longitudinal Study on Women s Health: sample. Available from https://www.alswh.org. au/about/sample. Accessed 25 Jan 2019

16. Navin Cristina TJ, Stewart Williams JA, Parkinson L, Sibbritt DW, Byles JE (2016) Identification of diabetes, heart disease, hypertension and stroke in mid- and older-aged women: Comparing self- 
report and administrative hospital data records. Geriatr Gerontol Int 16:95-102

17. Burton NW, Brown W, Dobson A (2010) Accuracy of body mass index estimated from self-reported height and weight in mid-aged Australian women. Aust N Z J Public Health 34(6):620-623. https://doi.org/10.1111/j.1753-6405.2010.00618.x

18. Curran PJ, Obeidat K, Losardo D (2010) Twelve Frequently Asked Questions About Growth Curve Modeling. J Cogn Dev 11(2):121136. https://doi.org/10.1080/15248371003699969

19. Nagin DS, Tremblay R (2005) Developmental trajectory groups: fact or a useful statistical fiction. Criminology 43:873-904

20. Tai MM (1994) A mathematical model for the determination of total area under glucose tolerance and other metabolic curves. Diabetes Care 17(2):152-154. https://doi.org/10.2337/diacare.17.2.152

21. Women s Health Australia (2019) Australian Longitudinal Study on Women s Health: Food frequency information / dietary questionnaire for epidemiology studies. Available from https://www.alswh. org.au/for-researchers/data/15-for-researchers/74-food-frequencyinformation. Accessed 5 Aug 2019

22. Muthen LK, Muthen BO (1998-2016) Mplus User's Guide (Seventh Edition). Muthen \& Muthen, Los Angeles, CA

23. Fagherazzi G, Vilier A, Affret A, Balkau B, Bonnet F, ClavelChapelon F (2015) The association of body shape trajectories over the life course with type 2 diabetes risk in adulthood: a group-based modeling approach. Ann Epidemiol 25(10):785-787. https://doi. org/10.1016/j.annepidem.2015.06.002

24. Mano Y, Yokomichi H, Suzuki K et al (2015) Do body mass index trajectories affect the risk of type 2 diabetes? A case-control study. BMC Public Health 15:718

25. Peter RS, Keller F, Klenk J, Concin H, Nagel G (2016) Body mass trajectories, diabetes mellitus, and mortality in a large cohort of Austrian adults. Medicine 95:e5608

26. Heianza Y, Arase Y, Kodama S et al (2015) Trajectory of body mass index before the development of type 2 diabetes in Japanese men: Toranomon Hospital Health Management Center Study 15. J Diabetes Investig 6(3):289-294. https://doi.org/10.1111/jdi.12308

27. Gordon-Larsen P, Koehler E, Howard AG et al (2014) Eighteen year weight trajectories and metabolic markers of diabetes in modernising China. Diabetologia 57:1820-1829

28. Thompson AL, Koehler E, Herring AH et al (2016) Weight gain trajectories associated with elevated C-reactive protein levels in Chinese adults. J Am Heart Assoc 5:e003262
29. Walsh EI, Shaw J, Cherbuin N (2018) Trajectories of BMI change impact glucose and insulin metabolism. Nutr Metab Cardiovasc Dis 28(3):243-251. https://doi.org/10.1016/j.numecd.2017.12.003

30. Liu R, Brickman WJ, Christoffel KK et al (2012) Association of adiposity trajectories with insulin sensitivity and glycemic deterioration: a longitudinal study of rural Chinese twin adults. Diabetes Care 35(7):1506-1512. https://doi.org/10.2337/dc11-2060

31. Zhang T, Xu J, Li S et al (2019) Trajectories of childhood BMI and adult diabetes: the Bogalusa Heart Study. Diabetologia 62:70-77

32. Zhang Y, Yatsuya H, Li Y et al (2017) Long-term weight-change slope, weight fluctuation and risk of type 2 diabetes mellitus in middle-aged Japanese men and women: findings of Aichi Workers' Cohort Study. Nutr Diabetes 7:e252

33. Hansen E, Hajri T, Abumrad NN (2006) Is all fat the same? The role of fat in the pathogenesis of the metabolic syndrome and type 2 diabetes mellitus. Surgery 139(6):711-716. https://doi.org/10.1016/ j.surg.2005.10.018

34. Kahn SE, Hull RL, Utzschneider KM (2006) Mechanisms linking obesity to insulin resistance and type 2 diabetes. Nature 444(7121): 840-846. https://doi.org/10.1038/nature05482

35. Felber JP, Golay A, Jequier E et al (1988) The metabolic consequences of long-term human obesity. Int J Obes 12(5):377-389

36. Tanamas SK, Wong E, Backholer K et al (2016) Age of onset of obesity and risk of type 2 diabetes. Aust N Z J Public Health 40(6): 579-581. https://doi.org/10.1111/1753-6405.12593

37. Moran A, Jacobs DR Jr, Steinberger J et al (1999) Insulin resistance during puberty: results from clamp studies in 357 children. Diabetes 48(10):2039-2044. https://doi.org/10.2337/diabetes.48.10.2039

38. The NS, Richardson AS, Gordon-Larsen P (2013) Timing and duration of obesity in relation to diabetes: findings from an ethnically diverse, nationally representative sample. Diabetes Care 36(4):865872. https://doi.org/10.2337/dc12-0536

39. Australian Institute of Health and Welfare (2019) Diabetes snapshot. Available from https://www.aihw.gov.au/reports/diabetes/ diabetes-snapshot/contents/how-many-australians-have-diabetes. Accessed 12 Jun 2019

Publisher's note Springer Nature remains neutral with regard to jurisdictional claims in published maps and institutional affiliations. 